\title{
2013 Wiener Prize
}

ANDREW J. MAJDA received the 2013 AMS-SIAM Norbert Wiener Prize in Applied Mathematics at the 119th Annual Meeting of the AMS in San Diego, California, in January 2013.

\section{Citation}

The 2013 Norbert Wiener Prize in Applied Mathematics is awarded to Andrew J. Majda for his

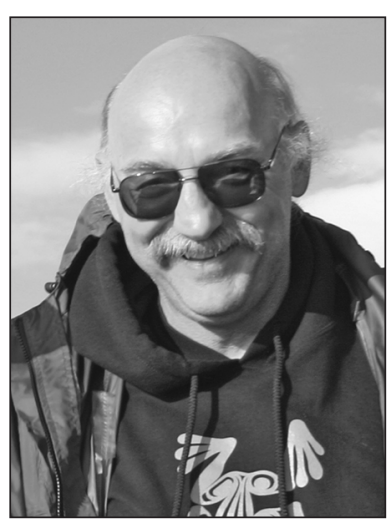
groundbreaking work in theoretical fluid mechanics and its application to problems in atmospheric science and oceanography. His many outstanding contributions to the field include his work on vortex dynamics, turbulent diffusion, concentration phenomena for the Euler equations, multidimensional shock fronts, and absorbing boundary conditions for wave propagation. Mathematicians and geophysicists alike have embraced Majda's pioneering advances on important and recalcitrant issues arising in cliAndrew J. Majda mate modeling and prediction. This work includes the development and exploitation of the methods of statistical physics in geophysical problems, as well as the multiscale analysis of moist fluid dynamics in the atmosphere.

\section{Biographical Sketch}

Andrew J. Majda is Morse Professor of Arts and Sciences at the Courant Institute of New York University. He was born in East Chicago, Indiana, on January 30, 1949. He received a B.S. degree from Purdue University in 1970 and a Ph.D. degree from Stanford University in 1973. Majda began his scientific career as a Courant Instructor at the Courant Institute from 1973-1975. Prior to returning to the Courant Institute in 1994, he held professorships at Princeton University (1984-1994), the University of California Berkeley (1978-1984), and the University of California Los Angeles (1976-1978).

Majda is a member of the National Academy of Sciences and the American Academy of Arts

DOI: http://dx.doi.org/10.1090/noti971 and Sciences and has received numerous honors and awards, including the National Academy of Science Prize in Applied Mathematics, the John von Neumann Prize of the Society of Industrial and Applied Mathematics, and the Gibbs Prize of the AMS. He has been awarded the Medal of the Collège de France twice, and he is a Fellow of the Japan Society for the Promotion of Science. Majda has received three honorary doctorates, including one from his undergraduate alma mater, Purdue University. He has given plenary one-hour lectures at both the ICM (Kyoto 1990) and the first ICIAM (Paris 1987) and is a fellow of both AMS and SIAM.

In his years at the Courant Institute, Majda has created the Center for Atmosphere Ocean Science with seven multidisciplinary faculty to promote cross-disciplinary research with modern applied mathematics in climate modeling and prediction.

\section{Response}

I am honored and delighted to receive the 2013 Norbert Wiener Prize from the American Mathematical Society and the Society for Industrial and Applied Mathematics. I believe in modern applied mathematics with the broadest possible toolkit blending asymptotic methods, numerical methods, and rigorous mathematical analysis with physical reasoning used to attack the most difficult and pressing scientific problems, such as climate modeling or turbulence. In this way, both a scientific discipline and mathematics can enrich each other in amazing ways. As a young scientist, I found I truly loved the serendipity between applied mathematics and complex physical phenomena, and I would like to thank Peter Lax and Joe Keller for being role models, in very different ways, during my early days at Courant. I would like to thank my many collaborators, Ph.D. students, and postdoctoral fellows for all of their insight, help, and friendship. For my cited earlier works, I would like to mention especially Tom Beale, Bjorn Engquist, and Ron DiPerna, a truly brilliant mathematician who left us too soon. For my cited work in climate atmosphere ocean science and applied mathematics, I would like to acknowledge Eric Vanden-Eijnden, Rupert Klein, Boualem 
Khouider, Sam Stechmann, and Dimitri Giannakis. Finally, I would like to thank my many friends and colleagues at the Courant Institute, especially David McLaughlin, for the wonderful, supportive atmosphere for genuine interdisciplinary research with mathematics and applications.

\section{About the Prize}

The Wiener Prize is awarded every three years to recognize outstanding contributions to applied mathematics in the highest and broadest sense (until 2001, the prize was awarded every five years). Established in 1967 in honor of Norbert Wiener (1894-1964), the prize was endowed by the Department of Mathematics of the Massachusetts Institute of Technology. The prize is given jointly by the AMS and the Society for Industrial and Applied Mathematics (SIAM). The recipient must be a member of one of these societies and a resident of the United States, Canada, or Mexico. The prize carries a cash award of US\$5,000.

The recipient of the Wiener Prize is chosen by a joint AMS-SIAM selection committee. For the 2013 prize, the members of the selection committee were: Jerry L. Bona, Roger Temam, and HorngTzer Yau.

The previous recipients of the Wiener Prize are: Richard E. Bellman (1970), Peter D. Lax (1975), Tosio Kato (1980), Gerald B. Whitham (1980), Clifford S. Gardner (1985), Michael Aizenman (1990), Jerrold E. Marsden (1990), Hermann Flaschka (1995), Ciprian Foias (1995), Alexandre J. Chorin (2000), Arthur T. Winfree (2000), James Sethian (2004), Craig Tracy and Harold Widom (2007), and David L. Donoho (2010).

- Elaine Kehoe

\section{San Diego, CA, Joint Mathematics Meetings Photo Key for pages 390-391}
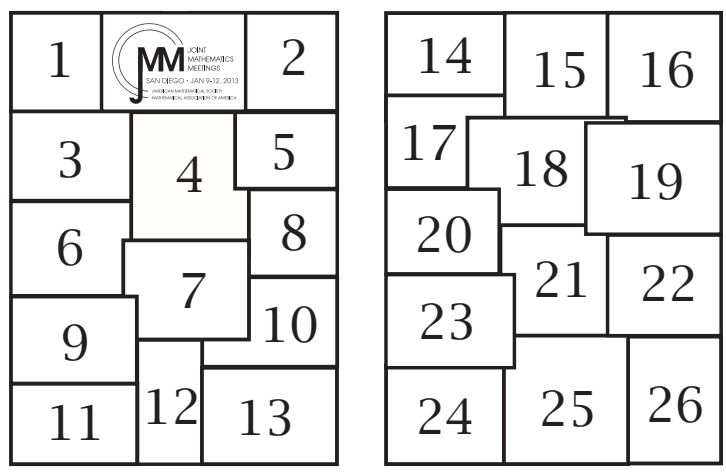

1. AMS Booth in the Exhibits area.

2. AMS Booth in the Exhibits area.

3. Contestants in Who Wants to Be a Mathematician National Game.

4. Coming down the escalator in the San Diego Convention Center.

5. Meeting and greeting in the AMS Booth.

6. Listening to a talk.

7. Mathematical Art Exhibit.

8. In the Exhibits area.

9. One of the winners, Mathematical Art Exhibit.

10. Email Center.

11. In the Exhibits area.

12. AMS Colloquium Lecture speaker Alice Guionnet.

13. In the Exhibits area.

14. Attentive audience.

15. Calvin Deng, winner of the San Diego Who Wants to Be a Mathematician Game.

16. Grad Student Fair.

17. Outside the San Diego Convention Center.

18. Undergraduate Student Poster Session.

19. Fellow students cheer on a Who Wants to Be a Mathematician contestant.

20. Invited Address audience.

21. I Love Math buttons.

22. Cédric Villani, AMS Josiah Willard Gibbs Lecture speaker.

23. Networking.

24. MAA Invited Address speaker Tony DeRose.

25. AMS-MAA Invited Address speaker Emily Shuckburgh.

26. AMS 125th Anniversary Gala, Saturday, January 12. 